\title{
Hiérarchie ou responsabilité par le diagnostic clinico-psychologique dans la psychothérapie?
}

\section{Une réflexion sur une perspective intégrée dans le cadre de la psychothérapie}

\author{
Angéla Szalontainé Krasznai
}

Psychotherapie-Wissenschaft 8 (1) 45-46 2018

www.psychotherapie-wissenschaft.info

CC BY-NC-ND

DOI: $10.30820 / 8242.08$

Mots-clés : diagnostic clinico-psychologique, diversité, authenticité, modèle, rôle, psychothérapie, identité de branches

Je vous prie de : Fermer les yeux et de plonger en vousmême. Observez ce qui se passe en vous, physiquement et mentalement, pendant que vous le faites. Si vous avez la sensation que vous pouvez en tant qu'être humain développer une relation à la personne assise devant vous avec des affections et des troubles, que vous pouvez devenir confiant et congruent par rapport à elle/lui et approfondir la connexion à elle/lui, faites-en une annotation mentale. Ouvrez alors les yeux, regardez la personne devant vous, et exprimez à votre manière les sentiments et la perception physique que vous avez sur vous-même et à l'encontre de l'autre personne. Et commencez à travailler dessus.

Dans ce processus, la première étape consiste à détecter et à accepter que chaque être humain que nous rencontrons est capable d'activer nos projections et, en raison des souvenirs, nos anciens stéréotypes. Lorsque nous pouvons faire la différence entre ce que nous voyons à trier dans nos tiroirs et que nous ne sommes pas toujours conscients que cela s'est passé. Lorsque nous l'avons décelé, nous pouvons commencer à nous occuper de la situation et à développer une différence précise entre les faits et nos opinions.

Il est pour moi important en tant que thérapeute de reconnaitre : Notre objectif doit être d'avoir consciemment et clairement devant les yeux que nous voyons toujours seulement ce que nous voyons et que cette image est une partie de nous et ne dit rien sur l'autre personne (par ex. cliente, patiente, ou personne avec des affections/troubles), lorsque nous regardons quelqu'un pour la première fois. «Absolument rien. » (Satir, 2011, p. 381) Cela peut être assimilé à un piège dans la pose de diagnostic, dans lequel le/la thérapeute essaie d'élaborer rapidement une description précise ou " exclusive » de visages et de la dynamique interne de certaines personnes, puis de considérer la partie comme la totalité.

Dans l'établissement clinique de diagnostic, l'objectivité thérapeutique est de mise. Selon moi, il est nécessaire qu'une acceptation thérapeutique de rôle soit consciemment possédée afin que nous évoluions vers une plus grande proportion de certitude, vers nos relations thérapeutiques plus intimes et vers plus d'attention de la personne avec des affections/troubles et envers les autres aussi.

La difficulté est dans ce travail interne : Aujourd'hui, au moins 400 différentes méthodes thérapeutiques sont utilisées dans le domaine clinique (Garfield \& Bergin, 1994 ; Karasu, 1992) et les possibilités de diagnostics ainsi que l'« inventaire méthodique » thérapeutique sont complétés par d'autres recherches et de nouveaux aspects et toujours et encore élargis. Ce qui a pour résultat qu'il existe une incroyable multitude, mais qui cache aussi le risque d'isolement de l'approche thérapeutique sélectionnée soi-même. Les questions centrales sont : "Comment je me positionne dans le processus thérapeutique ? "; "Comment je me définis et comment je définis ma responsabilité dans ce processus ? » et " quel est mon objectif de processus thérapeutique depuis la première rencontre avec la personne avec des affectations/troubles ?".

Certes, le thème actuel est l'établissement clinique de diagnostics, mais cela constitue déjà une première étape importante vers la thérapie qui peut avoir un effet favorable et/ou défavorable sur tout le déroulement de la thérapie. Dans cette première étape, il s'agit notamment de poser « la première pierre " en tant "qu'accompagnant, auxiliaire ou peut-être modèle ", et notamment exactement de la manière dont on se définit ou se caractérise soi-même en tant que thérapeute. Par ailleurs, l'identité thérapeutique est consciemment développée par l'intersubjectivité et la maturité de la personnalité. Ce cadre significatif défini individuellement de l'établissement de diagnostics et le rôle des thérapeutes dans la psychothérapie influencent la position de départ définie soi-même dans le contexte thérapeutique, et également le processus thérapeutique ultérieur.

Si un individu avec des troubles est considéré comme malade (patient(e)) ou traité même comme handicapé, cela a pour conséquence qu'il s'adapte consciemment ou inconsciemment à ce rôle et aux attentes qui y sont associées. Inconsciemment, ces personnes vont se structurer dans le rôle "inférieur, moins que toi " (Comer, 2005) et par la communication thérapeutique, la représentation que les thérapeutes ne veulent pas se confirme en 
se construisant sur ce modèle : " je ne suis pas OK, tu es OK »- Philosophie de vie (Berne, 2010). Ce qui peut faire naitre rapidement une position hiérarchique dans la relation thérapeutique, qui porte préjudice aux critères de la réciprocité.

Au final, mon interrogation reste sans réponse concrète. Cela incite toutefois à émettre quelques réflexions : Serait-il possible à un moment à l'aide d'une méthode intégrante de travailler à la fois sur l'auto-développement personnel et dans la psychothérapie et de prendre consciemment pour objectif les deux parties ensemble de la thérapie ? Déjà pendant le processus de l'établissement de diagnostics ? Indépendamment du statut : La personne avec des affections/troubles et la personne avec l'expertise sur les troubles physiques et organiques ainsi que psychiques agissent de la même manière.

Est-ce que cela reste une utopie du 21e siècle ou est-il possible de concrétiser que l'auto-responsabilité soit définie par un processus ouvert, dynamique basé sur la réciprocité, au lieu d'être définie par une vision polarisée qui a légitimement pour conséquence une autodétermination orientée vers le statut et le pouvoir de forcer les patients dans une hiérarchie ? Que le "trompe-l'œil » médical ou thérapeutique dans la position liée au travail repose plutôt sur l'humanité et déplace l'intégrité de l'accentuation d'exclusivité, chacun(e) peut le décider pour soi-même. Tous ont le choix et le potentiel de décision!

\section{L'auteure}

Angéla Szalontainé Krasznai, MSc, études à l'université ELTE Budapest (Hongrie), est psychothérapeute déléguée dans le cadre ambulatoire dans le domaine psychosomatique. Elle est membre des organisations et associations suivantes : FSP (Fédération suisse des psychologues), SSCP (Swiss Society for Coaching Psychlogy), ASMPP (Académie suisse de médecine psychosomatique et psychosociale), ICF (International Coach Federation), et membre extraordinaire de Systemis.ch (Association suisse de la thérapie et des conseils systémiques). Le point central de ses recherches est l'analyse du développement de la personnalité ainsi que la promotion de la base motivationnelle et la discipline.

\section{Contact}

Angéla Szalontainé Krasznai

Psychologue FSP

Waldeggstrasse 1

CH-9500 Wil

Suisse

E-mail : smileka@gmail.com 